\title{
Consumo de alimentos ultraprocessados por idosos frequentadores de uma clínica integrada de saúde em São Paulo
}

\section{Consumption of ultra-processed foods by older adults attending an health clinic in São Paulo}

\author{
Jéssica Natália Santos Melo, Aline Veroneze de Mello, \\ Hellen Daniela de Sousa Coelho
}

Como citar este artigo: MELO, JÉSSICA N. S.; MELLO, ALINE $V_{\text {.; }}$ COELHO, HELLEN D. S. Consumo de alimentos ultraprocessados por idosos frequentadores de uma clínica integrada de saúde em São Paulo. Revista Saúde (Sta. Maria). 2020; 46 ( I).

\section{Autor correspondente:}

Nome: Hellen Daniela de Sousa

Coelho

E-mail: hellencoelho@gmail.com

Formação Profissional: Doutora em

Saúde Pública pela Universidade

de São Paulo (USP), São Paulo,

SP, Brasil.

Filiação Institucional: Universidade Paulista

Endereço para correspondência:

Av. Torres de Oliveira, 330

Bairro: Jaguaré

Cidade: São Paulo

Estado: São Paulo

CEP: 05347-020

Data de Submissão:

19/12/2019

Data de aceite:

08/04/2020

Conflito de Interesse: Não há conflito de interesse

\section{(cc) $\mathrm{BY}-\mathrm{NC}-\mathrm{ND}$}

\section{RESUMO}

Objetivo: avaliar o consumo de alimentos ultraprocessados por idosos atendidos em uma clínica escola de saúde em São Paulo, considerando o seu estado nutricional, sexo e presença ou não de hipertensão. Métodos: Estudo transversal com 78 idosos ( $\geq 60$ anos de idade), de ambos os sexos, realizado com frequentadores de uma Clínica integrada de Saúde. Os dados foram coletados entre janeiro de 2013 e dezembro de 2018. Esta pesquisa faz parte do projeto de pesquisa temático denominado: "Avaliação Interdisciplinar da Saúde do idoso". As variáveis quantitativas foram testadas para normalidade pelo teste de Kolmogorov Smirnov, não apresentando normalidade. Portanto, diferenças entre as médias de consumo de ultraprocessados entre idosos e idosas, hipertensos e normotensos, eutróficos e sobrepeso, foram analisadas por teste Mann-Whitney $(p<0,05)$. Resultados: Identificou-se que $65,4 \%(n=51)$ dos indivíduos eram normotensos e $34,6 \%(n=27)$ eram hipertensos. Notou-se que os idosos apresentaram um maior consumo de refrigerante, hambúrguer e biscoito recheado do que as idosas, os eutróficos apresentaram um maior consumo de pão de forma/bisnaguinha do que os indivíduos com sobrepeso e os hipertensos apresentaram maior consumo de margarina quando comparados aos normotensos. Conclusão: As ações de prevenção de fatores de risco para as DCNT, como a hipertensão, além da promoção de hábitos de vida saudáveis focadas na redução do consumo de alimentos ultraprocessados, são componentes fundamentais para contribuir para uma melhor qualidade de vida nessa faixa etária, principalmente entre as mulheres idosas, que apresentaram maior prevalência de hipertensão e maior consumo de ultraprocessados como biscoito recheado, refrigerante e hambúrguer.

PALAVRAS-CHAVE: Idosos; Hipertensão arterial; Alimentos ultraprocessados.

\section{ABSTRACT}

Objective: To evaluate the consumption of ultra-processed foods by older adults patients seen at a health clinic in São Paulo, considering their nutritional status, sex and presence or absence of hypertension. Methods: Cross-sectional study with 78 older adults ( $\geq 60$ years old), of both sexes, carried out at an integrated health clinic. The data were collected between January 2013 and December 2018. This research is part of the thematic research project called: "Interdisciplinary assessment of the health of the elderly". As quantitative variables were tested for normality by the Kolmogorov Smirnov test, they did not show normality. Therefore, differences between the means of consumption of ultra-processed between the elderly and the elderly, hypertensive and normotensive, eutrophic and overweight, analyzed by the Mann-Whitney test ( $p$ $<0.05$ ). Results: It was identified that $65.4 \%(n=51)$ of individuals were normotensive and $34.6 \%(n=27)$ were hypertensive. It was noted that female older adults had a higher consumption of soft drinks, hamburgers and filled biscuits than male older adults, the eutrophics had a higher consumption of shaped bread/winkle than overweight individuals and hypertensive individuals had a higher consumption of margarine when compared to normotensive individuals. Conclusion: Actions to prevent risk factors for NCDs, such as hypertension, in addition to promoting healthy lifestyles focused on reducing the consumption of ultra-processed foods, are fundamental components to contribute to a better quality of life in this age group, especially among female older, who had a higher prevalence of hypertension and higher consumption of ultra-processed products such as sweet biscuits, soft drinks and hamburgers.

KEYWORDS: Older adults; Hypertension; Ultraprocessed foods. 


\section{INTRODUÇÃO}

No Brasil, entre os anos de 1950 e 2000, menos de $10 \%$ da população era constituída por idosos ${ }^{1}$. Estima-se que até 2070 a população idosa representará cerca de 35\% do total da população, ultrapassando crianças e jovens com até 29 anos, que, por outro lado, tiveram queda expressiva nos últimos anos ${ }^{1}$.

Nesta faixa etária também é comum o surgimento e/ou progressão de doenças crônicas não transmissíveis (DCNT), com maior destaque para a hipertensão arterial sistêmica (HAS), associada a alterações funcionais e/ou estruturais dos principais órgãos, como coração, rins, encéfalo e vasos sanguíneos ${ }^{2,3}$. A HAS ainda possui baixas taxas de controle, o que a torna um dos principais fatores de risco para as doenças cardiovasculares (DCV) e importante problema de saúde pública, já que atinge mais de $60 \%$ da população idosa e aumenta consideravelmente a mortalidade por DCV².

No controle da HAS as modificações no estilo de vida são de extrema importância, como exemplo do controle de peso, padrão alimentar adequado e saudável, redução de consumo de sódio, moderação no consumo de álcool e a prática de exercício físico². Dados da Pesquisa de Orçamentos Familiares - POF 2008/2009, indicaram que o consumo alimentar da população brasileira aponta inadequações, incluindo os idosos, especialmente com relação à ingestão de micronutrientes, em todas as regiões do país ${ }^{4,5}$. Menor qualidade da dieta dos idosos pode ocasionar importantes deficiências de nutrientes, além de contribuir para o desenvolvimento e progressão das DCNT ${ }^{4,5}$. Observa-se no Brasil um aumento no consumo de alimentos prontos para o consumo, industrializados, leite e cereais refinados, e um baixo consumo de frutas, verduras e legumes, grãos e cereais integrais em idosos 6,7,

Além disso, o panorama atual aponta um aumento exponencial no consumo de alimentos processados e ultraprocessados, que também têm sido relacionados ao desenvolvimento de doenças cardiovasculares ${ }^{9}$. Esses alimentos são geralmente ricos em calorias, açúcar, sódio e gordura, e pobre em fibras e micronutrientes, e por esse motivo, devem ser consumidos com cautela ${ }^{5,10,11}$. É importante ressaltar que o consumo de alimentos ultraprocessados está entre os principais fatores de risco para a HAS ${ }^{12,13}$.

Embora essas questões já tenham sido objeto de pesquisa no Brasil, poucos estudos se concentraram em identificar diferenças na qualidade da alimentação de idosos considerando o grau de processamento dos alimentos, segundo estado nutricional e presença de HAS, o principal fator de risco para as DCV. No entanto, esse conhecimento é importante para dar suporte específico e apropriado intervenções em saúde pública, bem como estabelecer comparações e fornecer informações sobre os aspectos da alimentação que precisam melhorar.

Considerando essas premissas, o presente estudo consiste em avaliar o consumo de alimentos ultraprocessados por idosos atendidos em uma clínica integrada de saúde em São Paulo, considerando o seu estado nutricional, sexo e presença ou não de hipertensão. 


\section{MÉTODO}

Trata-se de um estudo transversal, realizado em uma Clínica integrada de saúde da Universidade Paulista (UNIP), com indivíduos idosos ( $\geq 60$ anos de idade), de ambos os sexos. Os participantes do estudo aderiram voluntariamente à pesquisa, excluindo os indivíduos que apresentaram impossibilidade de participar da entrevista. Os dados foram coletados entre janeiro de 2013 e dezembro de 2018.

Esta pesquisa faz parte do projeto de pesquisa temático denominado: "Avaliação Interdisciplinar da Saúde do idoso", aprovado pelo Comitê de Ética em pesquisa pelo parecer no 43023/12. Todos os participantes do estudo receberam um Termo de Consentimento Livre e Esclarecido (TCLE) e em caso de anuência e assinatura foram incluídos no projeto.

Os dados deste estudo foram extraídos dos prontuários dos pacientes. Para caracterização da população utilizouse as seguintes variáveis: sexo; idade, Índice de Massa Corporal (IMC) e os níveis de pressão arterial para identificar se são normotensas ou hipertensas. Para as informações de consumo alimentar foi utilizado questionário de frequência alimentar (QFA), validado para idosos ${ }^{7}$. As respostas sobre as categorias de frequência de consumo de cada alimento ultraprocessado foram convertidas em porção média diária e a classificação baseada no Guia Alimentar da População Brasileira $(2014)^{10}$.

Para aferição do peso corporal foi utilizada uma balança com capacidade de peso de 150kg e graduação de 0,1 kg (TANITA® Arlington Heights, IL, EUA). Os idosos foram orientados a posicionarem-se em pé, no centro da balança, com o peso corporal igualmente distribuído entre os pés e roupas leves. Para a aferição da estatura foi utilizado 0 estadiômetro (Modelo professional Sanny, São Bernardo do Campo, Brasil) com capacidade de medição: 115 cm a 210 cm e tolerância: +/- 2 mm em 210 cm com resolução em milímetros $(\mathrm{mm})$ fixado a uma parede sem rodapés.

No momento da aferição os idosos foram orientados a estarem descalços, com os pés paralelos, eretos, com os braços relaxados ao longo do corpo e com cinco pontos encostados na parede, conforme plano de Frankfurt ${ }^{14}$. O IMC foi calculado de acordo com a seguinte fórmula IMC = peso $(\mathrm{kg}) /$ altura $^{2}(\mathrm{~m})$. Os pontos de corte de IMC adotados foram os preconizados por Lipschitz (1994)15, baixo peso (IMC < 22), eutrofia (IMC 22-27) e sobrepeso (IMC >27).

Para a análise de dados utilizou-se o Software Exce/ß 2016 e o Statistical Package for the Social Sciences (SSPS 2.0). A caracterização da população foi realizada por meio da distribuição de frequências absolutas e relativas para as variáveis qualitativas e médias para as variáveis quantitativas. As variáveis quantitativas foram testadas para normalidade pelo teste de Kolmogorov Smirnov, não apresentando normalidade. Portanto, diferenças entre as médias de consumo de ultraprocessados entre idosos, foram analisadas por teste Mann-Whitney, com nível descritivo p<0,05. 


\section{RESULTADO}

A amostra foi composta por 78 idosos, sendo $73 \%$ do sexo feminino e $27 \%$ do sexo masculino. Em relação à faixa etária $47,4 \%$ das idosas encontram-se entre 60 a 69 anos, e 25,6\% com 70 anos ou mais. Já entre os idosos, 12,9\% estavam na faixa etária entre 60 a 69 anos e 14,1\% possuíam 70 anos ou mais. De acordo com o estado nutricional o sexo feminino apresentou $28,2 \%$ com eutrofia e 44,9\% com sobrepeso, já o sexo masculino $11,5 \%$ com eutrofia e 15,4\% com sobrepeso. Em relação à classificação dos indivíduos em normotensos ou hipertensos, 47,4\% eram mulheres normotensas e 25,6\% hipertensas, enquanto os homens, 17,9\% são normotensos e 9,0\% eram hipertensos (Tabela 1). Tabela 1. Caracterização de idosos atendidos em uma clínica integrada de saúde em São Paulo segundo sexo. São Paulo, 2018.

\begin{tabular}{lllllll}
\hline VARIÁVEIS & \multicolumn{2}{c}{ Total } & \multicolumn{3}{c}{ Homens } & \multicolumn{2}{c}{ Mulheres } \\
\cline { 2 - 7 } & $\mathrm{n}$ & $\%$ & $\mathrm{n}$ & $\%$ & $\mathrm{n}$ & $\%$ \\
\hline $\begin{array}{l}\text { Sexo } \\
\text { Faixa etária (anos) }\end{array}$ & 78 & 100,0 & 21 & 27,0 & 57 & 73,0 \\
$\quad$ 60 a 69 & & & & & & \\
$\quad$ 70 ou mais & 31 & 39,7 & 11 & 14,1 & 20 & 25,6 \\
$\begin{array}{l}\text { Estado nutricional } \\
\quad \text { Eutrofia }\end{array}$ & 31 & 39,7 & 9 & 11,5 & 22 & 28,2 \\
$\quad$ Sobrepeso & 47 & 60,3 & 12 & 15,4 & 35 & 44,9 \\
Classificação da Pressão Arterial & & & & & & \\
$\quad$ Normotensão & 51 & 65,4 & 14 & 17,9 & 37 & 47,4 \\
$\quad$ Hipertensão & 27 & 34,6 & 7 & 9,0 & 20 & 25,6
\end{tabular}

No que se refere à frequência de consumo dos alimentos ultraprocessados entre os idosos, observou-se um alto consumo de margarina 17,9\% e 32,1\%, de 2 a 3 vezes/dia e 1 vez/dia, respectivamente, o que corresponde a cerca de metade da amostra estudada. Outro alimento que apresentou um consumo elevado foi o adoçante, com 25,6\% de 2 a 3 vezes/dia e 21,8\% 1 vez/dia. Foi verificado também o consumo frequente de macarrão instantâneo em parte da amostra, com $35,9 \% 1$ vez/semana e 15,4\% de 2 a 4 vezes/semana, mesmo resultado encontrado na frequência de 5 a 6 vezes/ semana. Molho de tomate foi o alimento com maior consumo $(41,0 \%)$, porém, numa frequência mais moderada de 1 vezl semana (Tabela 2).

Verificou-se menor consumo de temperos prontos (20,5\%) e biscoito salgado (15,4\%), 1 vez/dia, quando comparado à frequência de consumo nunca/menor que 1 vez/mês $(70,5 \%)$ nos temperos prontos e 35,9\% para biscoito salgado. Ressalta-se que o consumo de embutidos como mortadela/presunto e linguiça/salsicha, ambos com 16,7\% consumidos ao menos 1 vez/semana (Tabela 2). 
Tabela 2. Distribuição em número e percentual da frequência do consumo dos alimentos ultraprocessados. São Paulo, 2018.

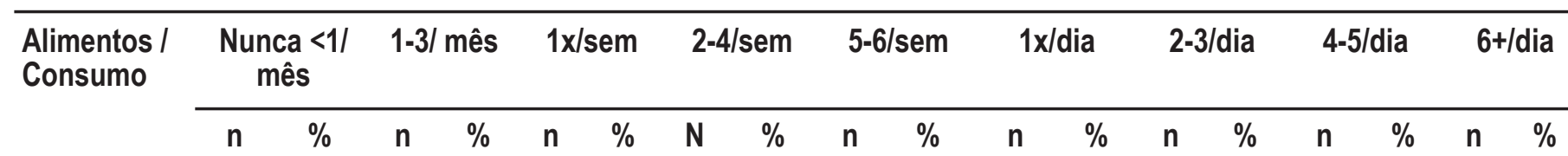

$\begin{array}{lllllllllllllllllll}\text { Creme de } & 58 & 74,4 & 14 & 17,9 & 3 & 3,8 & 3 & 3,8 & 0 & 0,0 & 0 & 0,0 & 0 & 0,0 & 0 & 0,0 & 0 & 0,0\end{array}$

Leite

$\begin{array}{lllllllllllllllllll}\text { Mortadela/ } & 31 & 39,7 & 23 & 29,5 & 13 & 16,7 & 7 & 9,0 & 0 & 0,0 & 3 & 3,8 & 1 & 1,3 & 0 & 0,0 & 0 & 0,0\end{array}$

Presunto

$\begin{array}{lllllllllllllllllll}\text { Linguiçal } & 36 & 46,2 & 20 & 25,6 & 13 & 16,7 & 6 & 7,7 & 0 & 0,0 & 2 & 2,6 & 1 & 1,3 & 0 & 0,0 & 0 & 0,0\end{array}$

Salsicha

Pão de

$\begin{array}{llllllllllllllllll}37 & 47,4 & 16 & 20,5 & 6 & 7,7 & 9 & 11,5 & 0 & 0,0 & 6 & 7,7 & 4 & 5,1 & 0 & 0,0 & 0 & 0,0\end{array}$

formal

Bisnaguinha

Cereal

Matinal

$\begin{array}{llllllllllllllllll}43 & 55,1 & 9 & 11,5 & 6 & 7,7 & 3 & 3,8 & 5 & 6,4 & 10 & 12,8 & 2 & 2,6 & 0 & 0,0 & 0 & 0,0\end{array}$

$\begin{array}{lllllllllllllllllll}\text { Macarrão } & 12 & 15,4 & 19 & 24,4 & 28 & 35,9 & 12 & 15,4 & 12 & 15,4 & 3 & 3,8 & 1 & 1,3 & 0 & 0,0 & 0 & 0,0\end{array}$ Instantâneo

$\begin{array}{lllllllllllllllllll}\text { Margarina } & 25 & 32,1 & 5 & 6,4 & 1 & 1,3 & 3 & 3,8 & 3 & 3,8 & 25 & 32,1 & 14 & 17,9 & 2 & 2,6 & 0 & 0,0\end{array}$

$\begin{array}{lllllllllllllllllll}\text { Chocolates } & 34 & 43,6 & 20 & 25,6 & 11 & 14,1 & 11 & 14,1 & 0 & 0,0 & 2 & 2,6 & 0 & 0,0 & 0 & 0,0 & 0 & 0,0\end{array}$

$\begin{array}{llllllllllllllllll}65 & 83,3 & 1 & 1,3 & 5 & 6,4 & 3 & 3,8 & 1 & 1,3 & 3 & 3,8 & 0 & 0,0 & 0 & 0,0 & 0 & 0,0\end{array}$

$\begin{array}{lllllllllllllllllll}\text { Sorvetes } & 36 & 46,2 & 25 & 32,1 & 9 & 11,5 & 5 & 6,4 & 2 & 2,6 & 0 & 0,0 & 1 & 1,3 & 0 & 0,0 & 0 & 0,0\end{array}$

$\begin{array}{lllllllllllllllllll}\text { Doces } & 58 & 74,4 & 9 & 11,5 & 6 & 7,7 & 3 & 3,8 & 0 & 0,0 & 2 & 2,6 & 0 & 0,0 & 0 & 0,0 & 0 & 0,0\end{array}$

$\begin{array}{lllllllllllllllllll}\text { Biscoito } & 28 & 35,9 & 9 & 11,5 & 12 & 15,4 & 10 & 12,8 & 2 & 2,6 & 12 & 15,4 & 5 & 6,4 & 0 & 0,0 & 0 & 0,0\end{array}$

Salgado

$\begin{array}{lllllllllllllllllll}\text { Biscoitos } & 40 & 51,3 & 12 & 15,4 & 9 & 11,5 & 5 & 6,4 & 3 & 3,8 & 9 & 11,5 & 0 & 0,0 & 0 & 0,0 & 0 & 0,0\end{array}$ doces sem

recheio

$\begin{array}{lllllllllllllllllll}\text { Biscoito } & 62 & 79,5 & 12 & 15,4 & 0 & 0,0 & 2 & 2,6 & 0 & 0,0 & 2 & 2,6 & 0 & 0,0 & 0 & 0,0 & 0 & 0,0\end{array}$

Recheado

$\begin{array}{lllllllllllllllllll}\text { Pão de } & 34 & 43,6 & 23 & 29,5 & 9 & 11,5 & 11 & 14,1 & 0 & 0,0 & 0 & 0,0 & 1 & 1,3 & 0 & 0,0 & 0 & 0,0\end{array}$

Queijo

$\begin{array}{lllllllllllllllllll}\text { Refrigerante } & 39 & 50,0 & 13 & 16,7 & 9 & 11,5 & 7 & 9,0 & 2 & 2,6 & 4 & 5,1 & 4 & 5,1 & 0 & 0,0 & 0 & 0,0\end{array}$

$\begin{array}{lllllllllllllllllll}\text { Suco } & 48 & 61,5 & 8 & 10,3 & 4 & 5,1 & 6 & 7,7 & 3 & 3,8 & 6 & 7,7 & 2 & 2,6 & 1 & 1,3 & 0 & 0,0\end{array}$

Artificial

$\begin{array}{lllllllllllllllllll}\text { Hambúrguer } & 58 & 74,4 & 16 & 20,5 & 3 & 3,8 & 1 & 1,3 & 0 & 0,0 & 0 & 0,0 & 0 & 0,0 & 0 & 0,0 & 0 & 0,0\end{array}$

$\begin{array}{lllllllllllllllllll}\text { Sopas } & 20 & 25,6 & 26 & 33,3 & 18 & 23,1 & 6 & 7,7 & 2 & 2,6 & 5 & 6,4 & 1 & 1,3 & 0 & 0,0 & 0 & 0,0\end{array}$

$\begin{array}{lllllllllllllllllll}\text { Molho } & 64 & 82,1 & 7 & 9,0 & 7 & 9,0 & 0 & 0,0 & 0 & 0,0 & 0 & 0,0 & 0 & 0,0 & 0 & 0,0 & 0 & 0,0\end{array}$

Maionese

$\begin{array}{lllllllllllllllllll}\text { Molhos } & 59 & 75,6 & 13 & 16,7 & 5 & 6,4 & 1 & 1,3 & 0 & 0,0 & 0 & 0,0 & 0 & 0,0 & 0 & 0,0 & 0 & 0,0\end{array}$ industriali-

zados

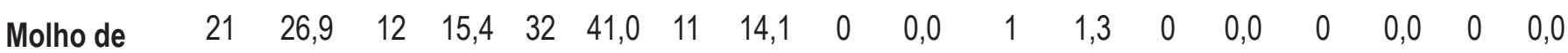
tomate

$\begin{array}{llllllllllllllllllll}\text { Adoçante } & 33 & 42,3 & 1 & 1,3 & 2 & 2,6 & 1 & 1,3 & 1 & 1,3 & 17 & 21,8 & 20 & 25,6 & 2 & 2,6 & 1 & 1,3\end{array}$

$\begin{array}{lllllllllllllllllll}\text { Temperos } & 55 & 70,5 & 1 & 1,3 & 5 & 6,4 & 1 & 1,3 & 0 & 0,0 & 16 & 20,5 & 0 & 0,0 & 0 & 0,0 & 0 & 0,0\end{array}$

Prontos 
De acordo com os resultados indicados na tabela 3, nota-se que os idosos apresentaram maior consumo de biscoito recheado $(p=0,004)$, refrigerante $(p=0,021)$ e hambúrguer $(p=0,025)$ do que as idosas, 0 que representa $12,5 \%$ dos alimentos ultraprocessados.

Tabela 3. Distribuição de média, desvio padrão e mediana do consumo de alimentos ultraprocessados entre homens e mulheres. São Paulo, 2018.

\begin{tabular}{|c|c|c|c|c|c|c|c|}
\hline \multirow[b]{2}{*}{ Alimentos } & \multicolumn{3}{|c|}{ Homens } & \multicolumn{3}{|c|}{ Mulheres } & \multirow[b]{2}{*}{$p^{*}$} \\
\hline & Média & $\begin{array}{l}\text { Desvio } \\
\text { Padrão }\end{array}$ & Mediana & Média & $\begin{array}{l}\text { Desvio } \\
\text { Padrão }\end{array}$ & Mediana & \\
\hline Creme de Leite & 0,0267 & 0,0888 & 0,0000 & 0,3277 & 0,0809 & 0,0000 & 0,182 \\
\hline Mortadela/Presunto & 0,2524 & 0,5630 & 0,0800 & 0,1146 & 0,2032 & 0,0800 & 0,341 \\
\hline Linguiça/Salsicha & 0,2210 & 0,5682 & 0,0800 & 0,0974 & 0,1656 & 0,0800 & 0,881 \\
\hline $\begin{array}{l}\text { Pão de forma/ Bisna- } \\
\text { guinha }\end{array}$ & 0,3514 & 0,7512 & 0,0800 & 0,2505 & 0,5208 & 0,0800 & 0,924 \\
\hline Cereal Matinal & 0,2843 & 0,6016 & 0,0000 & 0,2672 & 0,4740 & 0,0000 & 0,790 \\
\hline Macarrão Instantâneo & 0,2524 & 0,3232 & 0,1300 & 0,2151 & 0,3578 & 0,1300 & 0,935 \\
\hline Margarina & 1,1971 & 1,4942 & 0,7000 & 0,8367 & 0,8488 & 1,0000 & 0,815 \\
\hline Chocolates & 0,1457 & 0,1703 & 0,0800 & 0,1118 & 0,2043 & 0,0800 & 0,347 \\
\hline Achocolatado & 0,1171 & 0,2476 & 0,0000 & 0,0556 & 0,2058 & 0,0000 & 0,084 \\
\hline Sorvetes & 0,0976 & 0,1651 & 0,0800 & 0,1232 & 0,3467 & 0,0800 & 0,739 \\
\hline Doces & 0,0829 & 0,2298 & 0,0000 & 0,0519 & 0,1515 & 0,0000 & 0,988 \\
\hline Biscoito Salgado & 0,4424 & 0,7634 & 0,1300 & 0,4016 & 0,6143 & 0,1300 & 0,977 \\
\hline $\begin{array}{l}\text { Biscoitos doces sem } \\
\text { recheio }\end{array}$ & 0,2743 & 0,3960 & 0,0800 & 0,1661 & 0,3050 & 0,0000 & 0,185 \\
\hline Biscoito Recheado & 0,0648 & 0,1176 & 0,0000 & 0,0421 & 0,1857 & 0,0000 & $0,004^{*}$ \\
\hline Pão de Queijo & 0,1610 & 0,1782 & 0,0800 & 0,1146 & 0,3376 & 0,0800 & 0,143 \\
\hline Refrigerante & 0,5795 & 0,8676 & 0,1300 & 0,1446 & 0,3788 & 0,0000 & $0,021^{*}$ \\
\hline Suco Artificial & 0,1290 & 0,2724 & 0,0000 & 0,3237 & 0,7734 & 0,0000 & 0,282 \\
\hline Hambúrguer & 0,0414 & 0,0515 & 0,0000 & 0,0211 & 0,0596 & 0,0000 & $0,025^{\prime}$ \\
\hline Sopas & 0,1671 & 0,2899 & 0,0800 & 0,2142 & 0,3959 & 0,0800 & 0,345 \\
\hline Molho de Maionese & 0,0038 & 0,0175 & 0,0000 & 0,0244 & 0,0468 & 0,0000 & 0,060 \\
\hline $\begin{array}{l}\text { Molhos Industrializa- } \\
\text { dos }\end{array}$ & 0,0390 & 0,0934 & 0,0000 & 0,0223 & 0,0407 & 0,0000 & 0,875 \\
\hline Molho de Tomate & 0,1410 & 0,1385 & 0,1300 & 0,3256 & 1,4487 & 0,1300 & 0,854 \\
\hline Adoçante & 1,1762 & 1,1278 & 1,0000 & 1,0305 & 1,3639 & 0,4000 & 0,502 \\
\hline Temperos Prontos & 0,0990 & 0,3000 & 0,0000 & 0,264 & 0,4283 & 0,0000 & 0,071 \\
\hline
\end{tabular}

*teste Mann-Whitney - U nível descritivo $p<0,05$.

Observou-se também que os idosos eutróficos apresentaram um consumo do pão de forma/ bisnaguinha mais elevado $(p=0,048)$ do que os idosos com sobrepeso, representando $4,16 \%$ dos alimentos ultraprocessados do presente estudo (Tabela 4). 
Tabela 4. Distribuição de média, desvio padrão e mediana do consumo de alimentos ultraprocessados indivíduos com eutrofia e sobrepeso. São Paulo, 2018.

\begin{tabular}{|c|c|c|c|c|c|c|c|}
\hline & & Eutrofia & & & Sobrepeso & & \\
\hline Alimentos & Média & $\begin{array}{l}\text { Desvio } \\
\text { Padrão }\end{array}$ & Mediana & Média & $\begin{array}{l}\text { Desvio } \\
\text { Padrão }\end{array}$ & Mediana & $\mathrm{p}^{*}$ \\
\hline Creme de Leite & 0,5480 & 0,1211 & 0,0000 & 0,0215 & 0,0378 & 0,0000 & 0,693 \\
\hline Mortadela/Presunto & 0,1271 & 0,2070 & 0,0800 & 0,1679 & 0,4072 & 0,0800 & 0,910 \\
\hline Linguiça/Salsicha & 0,1142 & 0,2018 & 0,0800 & 0,1415 & 0,3898 & 0,0800 & 0,728 \\
\hline Pão de forma/ Bisnaguinha & 0,3181 & 0,5389 & 0,0800 & 0,2511 & 0,6226 & 0,0000 & $0,048^{*}$ \\
\hline Cereal Matinal & 0,2900 & 0,5469 & 0,0000 & 0,2598 & 0,4854 & 0,0000 & 0,626 \\
\hline Macarrão Instantâneo & 0,2581 & 0,4747 & 0,1300 & 0,2034 & 0,2315 & 0,1300 & 0,993 \\
\hline Margarina & 0,7642 & 0,8146 & 1,0000 & 1,0455 & 1,1943 & 1,0000 & 0,542 \\
\hline Chocolates & 0,1094 & 0,1398 & 0,0800 & 0,1285 & 0,2256 & 0,0800 & 0,627 \\
\hline Achocolatado & 0,0968 & 0,3005 & 0,0000 & 0,0560 & 0,1409 & 0,0000 & 0,274 \\
\hline Sorvetes & 0,1455 & 0,4488 & 0,0000 & 0,097 & 0,1615 & 0,0800 & 0,730 \\
\hline Doces & 0,0510 & 0,1806 & 0,0000 & 0,0664 & 0,1727 & 0,0000 & 0,540 \\
\hline Biscoito Salgado & 0,4681 & 0,6513 & 0,1300 & 0,376 & 0,6582 & 0,0800 & 0,265 \\
\hline Biscoito Maisena/Maria/Leite & 0,1797 & 0,2927 & 0,0800 & 0,2055 & 0,3595 & 0,0000 & 0,741 \\
\hline Biscoito Recheado & 0,0232 & 0,0750 & 0,0000 & 0,0647 & 0,2093 & 0,0000 & 0,416 \\
\hline Pão de Queijo & 0,1287 & 0,1550 & 0,0800 & 0,1260 & 0,3709 & 0,0800 & 0,201 \\
\hline Refrigerante & 0,2284 & 0,4735 & 0,0800 & 0,2936 & 0,6463 & 0,0000 & 0,166 \\
\hline Suco Artificial & 0,3984 & 0,9271 & 0,0000 & 0,1874 & 0,4409 & 0,0000 & 0,424 \\
\hline Hambúrguer & 0,0290 & 0,0443 & 0,0000 & 0,0249 & 0,0658 & 0,0000 & 0,918 \\
\hline Sopas & 0,2274 & 0,3258 & 0,0800 & 0,1845 & 0,3976 & 0,0800 & 0,657 \\
\hline Molho de Maionese & 0,0219 & 0,0467 & 0,0000 & 0,0168 & 0,0389 & 0,0000 & 0,714 \\
\hline Molhos Industrializados & 0,0197 & 0,0379 & 0,0000 & 0,0315 & 0,0701 & 0,0000 & 0,648 \\
\hline Molho de Tomate & 0,4794 & 1,9617 & 0,1300 & 0,1417 & 0,1366 & 0,1300 & 0,652 \\
\hline Adoçante & 0,7777 & 1,0195 & 0,0800 & 1,2623 & 1,4323 & 1,0000 & 0,187 \\
\hline Temperos Prontos & 0,3100 & 0,4548 & 0,0000 & 0,1600 & 0,3571 & 0,0000 & 0,129 \\
\hline
\end{tabular}

* teste Mann-Whitney- U nível de significância $p<0,05$.

Os idosos hipertensos apresentaram um maior consumo de margarina $(p=0,027)$ se comparados aos idosos normotensos, o que correspondeu a 4,16\% dos alimentos ultraprocessados do estudo (Tabela 5). 
Tabela 5. Distribuição de média, desvio padrão e mediana do consumo de alimentos ultraprocessados entre normotensos e hipertensos. São Paulo, 2018.

\begin{tabular}{|c|c|c|c|c|c|c|c|}
\hline \multirow[b]{2}{*}{ Alimentos } & \multicolumn{3}{|c|}{ Normotensos } & \multicolumn{3}{|c|}{ Hipertensos } & \multirow[b]{2}{*}{$\mathbf{p}^{*}$} \\
\hline & Média & $\begin{array}{l}\text { Desvio } \\
\text { Padrão }\end{array}$ & Mediana & Média & $\begin{array}{l}\text { Desvio } \\
\text { Padrão }\end{array}$ & Mediana & \\
\hline Creme de Leite & 0,0380 & 0,0978 & 0,0000 & 0,0285 & 0,0421 & 0,0000 & 0,390 \\
\hline Mortadela/Presunto & 0,1904 & 0,4139 & 0,0800 & 0,0785 & 0,0777 & 0,0800 & 0,952 \\
\hline Linguiça/Salsicha & 0,1620 & 0,3976 & 0,0000 & 0,0715 & 0,0825 & 0,0800 & 0,845 \\
\hline Pão de forma/ Bisnaguinha & 0,3308 & 0,6342 & 0,0800 & 0,1774 & 0,4845 & 0,0000 & 0,298 \\
\hline Cereal Matinal & 0,2880 & 0,5682 & 0,0000 & 0,2411 & 0,3745 & 0,0800 & 0,548 \\
\hline Macarrão Instantâneo & 0,2437 & 0,4104 & 0,1300 & 0,1900 & 0,1766 & 0,1300 & 0,991 \\
\hline Margarina & 0,7792 & 0,9968 & 0,7000 & 1,2256 & 1,1401 & 1,0000 & $0,027^{*}$ \\
\hline Chocolates & 0,1065 & 0,1783 & 0,0800 & 0,1481 & 0,2248 & 0,0800 & 0,467 \\
\hline Achocolatado & 0,0733 & 0,2419 & 0,0000 & 0,0700 & 0,1674 & 0,0000 & 0,378 \\
\hline Sorvetes & 0,0986 & 0,1632 & 0,0800 & 0,1496 & 0,4768 & 0,0800 & 0,800 \\
\hline Doces & 0,0788 & 0,2117 & 0,0000 & 0,0252 & 0,0493 & 0,0000 & 0,593 \\
\hline Biscoito Salgado & 0,4737 & 0,7022 & 0,1300 & 0,2970 & 0,5409 & 0,0800 & 0,352 \\
\hline $\begin{array}{l}\text { Biscoito Maisena/Maria/ } \\
\text { Leite }\end{array}$ & 0,2151 & 0,3535 & 0,0000 & 0,1570 & 0,2919 & 0,0000 & 0,630 \\
\hline Biscoito Recheado & 0,0596 & 0,2015 & 0,0000 & 0,0267 & 0,0800 & 0,0000 & 0,720 \\
\hline Pão de Queijo & 0,1584 & 0,3670 & 0,0800 & 0,0678 & 0,0808 & 0,0800 & 0,692 \\
\hline Refrigerante & 0,2914 & 0,6166 & 0,0000 & 0,2056 & 0,5135 & 0,0800 & 0,735 \\
\hline Suco Artificial & 0,3271 & 0,7533 & 0,0000 & 0,1659 & 0,5086 & 0,0000 & 0,302 \\
\hline Hambúrguer & 0,0292 & 0,0648 & 0,0000 & 0,0215 & 0,0425 & 0,0000 & 0,689 \\
\hline Sopas & 0,2220 & 0,4247 & 0,0800 & 0,1630 & 0,2330 & 0,0800 & 0,918 \\
\hline Molho de Maionese & 0,0231 & 0,0461 & 0,0000 & 0,0107 & 0,0319 & 0,0000 & 0,235 \\
\hline Molhos Industrializados & 0,0280 & 0,0666 & 0,0000 & 0,0244 & 0,0437 & 0,0000 & 0,850 \\
\hline Molho de Tomate & 0,3494 & 1,5307 & 0,1300 & 0,137 & 0,1378 & 0,1300 & 0,982 \\
\hline Adoçante & 1,0773 & 1,3096 & 1,0000 & 1,0556 & 1,3034 & 0,0000 & 0,657 \\
\hline Temperos Prontos & 0,2498 & 0,4247 & 0,0000 & 0,1626 & 0,3583 & 0,0000 & 0,533 \\
\hline
\end{tabular}

* teste Mann-Whitney - U nível descritivo $p<0,05$.

\section{DISCUSSÃO}

No presente estudo houve uma maior prevalência de idosas que buscaram assistência à saúde, de idosos na faixa etária de 60 a 69 anos, com sobrepeso, normotensos e maior prevalência de hipertensão entre as mulheres.

Verificou-se o consumo diário de adoçante de 25,6\% com frequência de 2 a 3 vezes/dia e 21,8\% uma vez/dia. O uso de adoçante é recomendado para pessoas diabéticas, porém o presente estudo não avaliou quantos idosos eram diabéticos. No ano de 2003 a população diabética era aproximadamente de 150 milhões, e estima-se que esse número dobre até 0 ano de $2025^{16}$. 
O consumo de embutidos como mortadela/presunto e linguiça/salsicha, representaram 16,7\% da amostra, com consumo de ao menos 1 vez/semana, sendo que a maioria dos idosos consumiam nunca/menos de 1 vez/mês, isto é, $39,7 \%$ e 46,2\%, respectivamente. Diferentemente do resultado obtido no estudo de Gadenz e Benvegnú $(2013)^{18}$, que verificou um alto consumo de embutidos entre os idosos entrevistados $(63,2 \%)$.

Observou-se menor consumo de temperos prontos $(20,5 \%)$ e biscoito salgado $(15,4 \%)$ na frequência de 1 vezl dia. Porém, identificou-se resultados diferentes aos da literatura. Previato et al. (2015) ${ }^{17}$, que avaliaram o perfil clíniconutricional e alimentar de idosos participantes do Programa da Terceira Idade Vitalidade e Cidadania, encontrando uma ingestão elevada de alimentos ultraprocessados (93\%), sendo que $89 \%$ dos idosos relataram consumir biscoitos e guloseimas diariamente, como também no estudo de Gadenz e Benvegnú $(2013)^{18}$, no qual foram analisados os hábitos alimentares de idosos hipertensos, sendo que $68,4 \%$ dos entrevistados relataram o uso de temperos industrializados. Isso pode ser explicado, pois no presente estudo os participantes foram constituídos de idosos frequentadores de uma Clínica Integrada de Saúde, e que possivelmente, devido às orientações profissionais anteriores, já tenham maior consciência e preocupação com seus hábitos alimentares.

Observou-se que o aumento do consumo de biscoitos salgados, vem crescendo devido à sua praticidade e maior prazo de validade quando comparados com alimentos in natura e minimamente processados. Em estudo realizado por Costa, Gonçalves e Oliveira $(2013)^{19}$, que analisou o teor de sódio em 106 rótulos de produtos industrializados, observaram-se teores elevados de sódio no biscoito cream cracker água e sal.

A falta de conhecimento quanto ao processamento dos alimentos, tais como diferenças, por exemplo, entre um macarrão instantâneo e massa alimentícia tradicional ou molho de tomate caseiro e industrializado, pode explicar o consumo elevado de macarrão instantâneo, sendo que 35,9\% dos idosos relataram consumir 1 vez/semana e 15,4\% de 2 a 4 vezes/semana, assim como de 5 a 6 vezes/semana. Assim como de molho de tomate industrializado, sendo consumido pelo menos 1 vez por semana, com um percentual de 41,0\%. Um estudo realizado por Candeias e Amaral $(2016)^{20}$, com mulheres de 16 a 61 anos beneficiárias do Programa Bolsa Família, que avaliou a frequência de consumo dos marcadores de alimentação saudável e não saudável, verificou maiores frequências de consumo de macarrão instantâneo, salgadinho de pacote e biscoito salgado em 51,80\% da população estudada. Já Previato et al. (2015) ${ }^{17}$, que avaliaram o perfil clínico-nutricional e alimentar de 28 idosos, mostrou alta prevalência de idosos que consumiam molhos e massas (93\%).

Ao relacionar o consumo de alimentos ultraprocessados com sexo, foi verificado que os idosos do sexo masculino apresentaram um maior consumo de biscoito recheado, refrigerante e hambúrguer do que os idosos do sexo feminino, o que condiz com o consumo alimentar da população, na qual geralmente, homens tem hábitos alimentares mais inadequados, quando comparados com mulheres. No estudo realizado por Destri, Zanini e Assunção (2017) ${ }^{21}$ que avaliou a prevalência de consumo alimentar entre hipertensos e diabéticos na cidade de Nova Boa Vista-RS, encontrou 
uma maior prevalência de consumo de refrigerante no sexo feminino. Já o estudo de Orlando et al. $(2010)^{22}$, realizado em um município do norte do Rio Grande do Sul, que visava avaliar a qualidade da alimentação e sua relação com as DCNT num grupo de idosos entre 60 e 85 anos, encontrou consumo diário de refrigerantes em $61 \%$ dos idosos. Produtos como refrigerantes, biscoitos, bolos, entre outros, apresentam alto teor de sódio, gorduras e açúcares, sendo pobres em nutrientes essenciais ${ }^{23}$.

Quanto ao consumo dos ultraprocessados com relação ao estado nutricional dos idosos, apenas o pão de forma/ bisnaguinha apresentou diferença significativa, sendo que os idosos eutróficos apresentaram um consumo maior do que idosos com sobrepeso, o que pode ser explicado pelo tamanho amostral e a causalidade reversa intrínseca aos estudos transversais. O consumo elevado de carboidratos refinados é comumente relacionado com o aumento da incidência de sobrepeso e obesidade $\mathrm{e}^{24,25}$.

No presente estudo foi encontrado um alto consumo de margarina de 2 a 3 vezes/dia (17,9\%) e 1 vez/dia $(32,1 \%)$. Ao relacionar o consumo de alimentos ultraprocessados entre normotensos e hipertensos, verificou-se um maior consumo de margarina pelos idosos hipertensos do que os normotensos. Em estudo na cidade de Nova Boa Vista-RS, utilizando-se o questionário do Sistema de Vigilância Alimentar e Nutricional (SISVAN), com adultos e idosos hipertensos e diabéticos, foi encontrado um consumo considerável de salada crua $(60,7 \%)$, frutas $(73,0 \%)$, lácteos $(61,4 \%)$, frituras $(57,3 \%)$, salgados $(66,4 \%)$ e refrigerantes $(76,3 \%)$; embutidos e doces foram os alimentos que tiveram maior frequência de consumo não recomendado, $34,8 \%$ e $23,9 \%$ respectivamente ${ }^{26}$. Vale ressaltar que os idosos foram entrevistados antes de receberem qualquer orientação nutricional dos profissionais da clínica integrada de saúde. Para a prevenção primaria da HAS, é de extrema importância a adoção de hábitos alimentares saudáveis como reduzir o consumo de alimentos ricos em sódio e gordura ${ }^{27}$. Menos de $10 \%$ dos idosos brasileiros possuem uma dieta adequada, necessitando de modificações na alimenาtação para atender suas necessidades nutricio $n$ nais. Devido ao desenvolvimento das DCNT estar frequentemente relacionado com hábitos de vida não saudáveis, medidas de prevenção que promovam boas práticas alimentares, são fundamentais para evitá-las ${ }^{28}$.

\section{CONSIDERAÇÕES FINAIS}

Considerando os resultados obtidos neste estudo, apesar dos idosos apresentarem uma frequência considerável de consumo diário e de 5 a 6 vezes/semana de alimentos ultraprocessados, a maior parte dos indivíduos possuíam bons hábitos alimentares, uma vez que as quantidades e frequência de consumo nunca $/<1$ vez/mês dos alimentos ultraprocessados foram superiores. Contudo, ações de prevenção dos fatores de risco para as DCNT, como a hipertensão, e a promoção de hábitos de vida saudáveis focadas na redução do consumo de alimentos ultraprocessados, são 
componentes fundamentais para contribuir para uma melhor qualidade de vida nessa faixa etária, principalmente entre as mulheres idosas, que apresentaram maior prevalência de hipertensão e maior consumo de ultraprocessados como biscoito recheado, refrigerante e hambúrguer.

\section{REFERÊNCIAS}

1. Instituto Brasileiro de Geografia e Estatística. Síntese de indicadores sociais: Uma análise das condições de vida da população brasileira. Rio de Janeiro: Estudos e Pesquisas Informação Demográfica e Socioeconômica; n.36, 2016.

2. Sociedade Brasileira de Cardiologia. $7^{\text {a }}$ Diretriz Brasileira de Hipertensão. Arq Bras Cardiol; 2016; 107(3 supl.3): 1-103.

3. Dutra DD, Duarte MCD, Albuquerque KF, Lima AS, Santos JS, Souto HC. Doenças cardiovasculares e fatores associados em adultos e idosos cadastrados em uma unidade básica de saúde. Rev Online Pesq. 2016; 8(2):4501-4509.

4. Nunes PMF. Avaliação da Ingestão de nutrientes, segundo padrão alimentar de idosos do município de Botucatu - SP. Tese (doutorado) - Universidade Estadual Paulista "Júlio de Mesquita Filho", Faculdade de Medicina de Botucatu, 2015.

5. Louzada MLC, Martins APB, Canella DS, Baraldi LG, Levy RB, Claro RM, et al. Alimentos ultraprocessados e perfil nutricional da dieta no Brasil. Rev. Saúde Pública 2015; 49:38.

6. Geib LTC. Determinantes sociais da saúde do idoso. Ciênc. saúde coletiva. 2012; 17(1): 123-133.

7. Viebig RF. Consumo de frutas e hortaliças e funcionamento cognitivo em idosos. São Paulo. Tese (doutorado) - Faculdade de Medicina da Universidade de São Paulo. Programa de Medicina Preventiva, 2010.

8. Souza AM, Pereira RA, Yokoo EM, Levy RB, Sichieri R. Alimentos mais consumidos no Brasil: Inquérito Nacional de Alimentação 2008-2009. Rev. Saúde Pública. 2013; 47 (Suppl 1): 190s-199s. 
9. Louzada MLDC, Martins APB, Canella DS, Baraldi LG, Levy RB, Claro RM, et al. Alimentos ultraprocessados e perfil nutricional da dieta no Brasil. Rev. Saúde Pública. 2015; 49(38): 01-11.

10. Brasil. Guia alimentar para a população brasileira. Ministério da Saúde, Secretaria de Atenção à Saúde, Departamento de Atenção Básica. - 2. ed. - Brasília: Ministério da Saúde, 2014. 156 p.: il. ISBN 978-85-334-2176-9.

11. Bielemann RM, Motta JVS, Minten GC, Horta BL, Gigante DP. Consumo de alimentos ultraprocessados e impacto na dieta de adultos jovens. Rev. Saúde Pública. 2015; 49: 28.

12. Monteiro CA, Cannon G, Moubarac JC, Levy RB, Louzada MLC, Jaime PC. The UN Decade of Nutrition, the NOVA food classification and the trouble with ultra-processing. Public Health Nutrition, 2018, 21(1), 5-17.

13. Rodrigues LG, Teodoro AJ. Redução de sódio em alimentos processados e ultraprocessados: impactos na produção de alimentos e na saúde pública. Rev Hipertens. 2015, 18(3): 37-45.

14. Lanpop - Laboratório de Avaliação Nutricional de Populações. Manual de técnicas antropométricas. São Paulo: Faculdade de Saúde Pública da USP; 2006.

15. Lipschitz DA. Screening for nutritional status in the eldery. PrimaryCarw. 1994, 21(1):55-67.

16. Cotta RMM, Reis RS, Batista KCS, Dias G, Alfenas RCG, Castro FAF. Hábitos e práticas alimentares de hipertensos e diabéticos: repensando o cuidado a partir da atenção primária. Rev. Nutr. 2009; 22(6): 823-835.

17. Previato HDRA, Barros FSS, Mello JBM, Silva FCS, Nimer M. Perfil clínico-nutricional e consumo alimentar de idosos do Programa Terceira Idade, Ouro Preto-MG. Demetra. 2015, 10(2): 375-387.

18. Gadenz SD, Benvegnú LA. Hábitos alimentares na prevenção de doenças cardiovasculares e fatores associados em idosos hipertensos. Ciênc. saúde coletiva. 2013; 18(12): 3523-3533.

19. Souza ADM, Souza BDSND, Bezerra IN, Sichieri R. Impacto da redução do teor de sódio em alimentos 
processados no consumo de sódio no Brasil. Cad Saúde Pública, 2016, 32, e00064615.

20. Candeias VL, Amaral JS. Avaliação do consumo alimentar das mulheres atendidas pelo Programa Bolsa Família do Município de Rondolândia-MT. XIV Jornada Científica das Faculdades Integradas de Cacoal - UNESC; 2016.

21. Destri K, Zanini RV, Assunção MCF. Prevalência de consumo alimentar entre hipertensos e diabéticos na cidade de Nova Boa Vista, Rio Grande do Sul, Brasil, 2013. Epidemiol. Serv. Saúde 2017; 26(4): 857-868.

22. Orlando $\mathrm{R}$, et al. Avaliação da alimentação e sua relação com as doenças crônicas não transmissíveis de um grupo de idosos de um município da região norte do estado do RS. Rev Enferm - Frederico Westphalen. 2011, 6(7):203-217.

23. Teixeira GLSB, Farias DMA, Macário YC, Silva AN, Vieira RS, Jadiel da Silva Nascimento. Teores de sódio, Açúcares e Lipídeos de Alimentos Infantis Comercializados no Município de Caruaru - PE. 2015; 1(1).

24. Bortoli C, Bonatto S, Bruscato NM, Siviero J. Ingestão dietética de gordura saturada e carboidratos em adultos e idosos com dislipidemias oriundos do Projeto Veranópolis. Rev Bras Cardiol. 2011; 24(1):33-41.

25. Martins M.V, Souza JD, Franco FS, Martinho KO, Tinoco ALA, et al. Consumo alimentar de idosos e sua associação com o estado nutricional. HU Revista. 2016, 42(2): 125-131.

26. Destri K, Zanini RDV, Assunção MCF. Prevalência de consumo alimentar entre hipertensos e diabéticos na cidade de Nova Boa Vista, Rio Grande do Sul, Brasil, 2013. Epidemiol Serv Saúde. 2017; 26(1): 857-868.

27. Silva YMS, Ramos RJ, Acioly PL. Avaliação do consumo alimentar de um grupo de idosos hipertensos do bairro bela vista de São José/SC. Rev Eletrônica Estácio Saúde. 2014; 3(1).

28. Gomes AP, Soares ALG, Gonçalves H. Baixa qualidade da dieta de idosos: estudo de base populacional no sul do Brasil. Ciênc. saúde coletiva. 2016; 21(11): 3417-3428. 